7 Tedford RJ, Beaty CA, Mathai SC, et al. Prognostic value of the pre-transplant diastolic pulmonary artery pressure-to-pulmonary capillary wedge pressure gradient in cardiac transplant recipients with pulmonary hypertension. J Heart Lung Transplant 2014; 33: 289-297.

8 Tampakakis E, Leary PJ, Selby VN, et al. The diastolic pulmonary gradient does not predict survival in patients with pulmonary hypertension due to left heart disease. JACC Heart Fail 2015; 3: 9-16.

9 Dragu R, Rispler S, Habib M, et al. Pulmonary arterial capacitance in patients with heart failure and reactive pulmonary hypertension. Eur J Heart Fail 2015; 17: 74-80.

10 Ibe T, Wada H, Sakakura K, et al. Pulmonary hypertension due to left heart disease: The prognostic implications of diastolic pulmonary vascular pressure gradient. J Cardiol 2016; 67: 555-559.

11 O'Sullivan CJ, Wenaweser P, Ceylan O, et al. Effect of pulmonary hypertension hemodynamic presentation on clinical outcomes in patients with severe symptomatic aortic valve stenosis undergoing transcatheter aortic valve implantation: insights from the new proposed pulmonary hypertension classification. Circ Cardiovasc Interv 2015; 8: e002358.

12 Nichols EL, Rezaee ME, Brown JR. One-year survival in heart failure patients with preserved ejection fraction and isolated post-capillary or combined post- and pre-capillary pulmonary hypertension. Circulation 2015; 132: Suppl. 3, A14302.

13 Gerges M, Gerges C, Pistritto AMA, et al. Pulmonary hypertension in heart failure: epidemiology, right ventricular function and survival. Am J Respir Crit Care Med 2015; 192: 1234-1246.

14 Miller WL, Grill DE, Borlaug BA. Clinical features, hemodynamics, and outcomes of pulmonary hypertension due to chronic heart failure with reduced ejection fraction: pulmonary hypertension and heart failure. JACC Heart Fail 2013; 1: 290-299.

\title{
A retrospective cohort study of patients with pulmonary embolism: the impact of comorbidities on patient's outcome
}

\author{
To the Editor:
}

Despite considerable advances in diagnosis and treatment, pulmonary embolism (PE) remains is an important clinical entity with a high risk of death. Due to pulmonary bed obstruction, PE can result in acute right ventricular failure, a life-threatening condition.

If untreated, acute PE is associated with a significant mortality rate (as high as 30\%) whereas the casefatality ratio of diagnosed and treated PE drops to $8 \%$ [1]. It is well known that comorbidities can affect one or more diseases through several potential aetiological mechanisms of direct causation, associated risk factors, heterogeneity and independence. In particular, in patients with venous thromboembolism (VTE) the presence of comorbidities can hinder clinical assessment and timely diagnoses [2] and exacerbate the risk of recurrence, complications and death [3].

In order to identify the burden of $\mathrm{PE}$, as well as the most common diagnoses associated with hospital admissions for PE, we analysed the hospitalisation data available for Sardinia (Italy) between 2009 and 2011. The secondary aim was to analyse the difference in comorbidity rates between survivors and patients who died. The study was conducted by analysing data from hospital discharge records (HDR; SDO in Italian) featuring the International Classification of Diseases, ninth revision, Clinical Modification (ICD-9-CM) code for PE (415. 1x) over the period 2009-2011 in Sardinia. Data were obtained from the Department of Health and Hygiene of the "Regione Autonoma della Sardegna" (RAS) Sardinian Regional Government. The HDR includes information pertaining to the hospitalisation and discharge of patients, both resident and non-resident in Sardinia, from all public and private hospitals located in this Italian region. All hospitals are required to provide the Department of Health and Hygiene at RAS with this information in electronic format. Information contained in the HDR is encoded according to the ICD-9-CM. It includes patient demographic characteristics, the field of primary diagnosis (defined as the main reason for hospitalisation), up to six fields for secondary diagnoses, hospital identification codes and diagnostic procedures. The Regional Epidemiological Observatory is a Division of the Department of Health and Hygiene at RAS devoted, among other things, to the management of healthcare information for epidemiological purposes.

Data were processed and descriptive statistics provided using MedCalc software (version 13.3.0.0; Ostend, Belgium). Analysis comprised all hospitalisations with $\mathrm{PE}$ as a primary hospital discharge diagnosis from 2009 to 2011. Data were expressed as absolute numbers, percentage and mean \pm SD. The rates were standardised to the Sardinian population between 2009 and 2011. 
Comorbidities in discharge records were identified using ICD-9-CM codes. Regression analysis was carried out to calculate the impact of comorbidities on PE mortality by means of odds ratios and $95 \%$ confidence intervals using death as the dependent variable. The following were included as comorbidities: old age ( $>75$ years), sex, blood hypertension, previous stroke, chronic obstructive pulmonary disease (COPD), diabetes mellitus, atrial fibrillation, cancer, deep vein thrombosis (DVT) and heart failure.

According to the National Institute of Statistics, the average annual Sardinian population between 2009 and 2011 was 1.672 million, ranging from 1.671 million in 2009 to 1.675 million in 2011, with 19020 hospitalisations for any cause per 100000 inhabitants per year (19\%). On average, there were 318018 hospitalisations per year, ranging from 300920 to 335115 , with a total of 954054 hospitalisations between 2009 and 2011. A total of 971 PE cases (primary diagnosis) were identified, with 101.8 discharges with a PE diagnosis per 100000 all-cause hospitalisations. The annual rate of discharges with a PE diagnosis in 2009 was $322 \mathrm{PE}$ discharges, i.e. 19.2 PE discharges per 100000 inhabitants per year. These rates were similar in 2010 and 2011. Overall, 614 (63.2\%) were women and the mean age was 71.5 years. The peak age was between 66 and 85 years. The overall average annual hospitalisation rate for PE was 0.11 (95\% CI 0.10-0.13) per 100000 individuals. This rate was similar during the 3 years of the study period: $322(0.11)$ in 2009, 353 (0.12) in 2010 and $346(0.12)$ in 2011. The procedure codes for diagnostic tests were available for 608 (62.6\%) out of the 971 cases with PE diagnosis. Computed tomography pulmonary angiography (CTPA) had been used in 567 (58.4\%) patients, ventilation/perfusion lung scanning in 27 (2.8\%) and arteriography in 17 (1.7\%) (three patients underwent both CTPA and lung scan). This information was not provided for the remaining 363 patients. We, therefore, decided to analyse the smaller cohort of patients for whom the final diagnosis of PE appeared properly proven. Between 2009 and 2011, 58 (9.54\%) out of 608 subjects with PE died during hospitalisation. The final logistic regression model included four variables (age $>75$ years, high blood pressure, DVT and atrial fibrillation) and the interaction between blood hypertension and atrial fibrillation. The other variables and interactions were not significantly associated with PE-related death, and were, therefore, excluded from the model. The Hosmer-Lemeshow test $(\mathrm{p}=0.88)$ indicates a good logistic regression model fit (table 1$)$. The results showed that age $>75$ years and previous ischaemic stroke are independent risk factors for mortality from PE. This figure does not appear surprising, as it has already reported.

We suggest a possible protective effect against mortality from PE, namely, the concomitance of DVT and of blood hypertension. For the former, our findings are consistent with those reported by WEBEROVA et al. [4], in 2012, who found a higher prevalence of DVT in survivors of embolic events. Possible explanations include the smaller burden of thrombus dislodgement in fragments coming from DVT, and the implementation of anticoagulation in patients with DVT, which, in turn, may have reduced the amount of fibrin detached from the thrombus. However, another hypothesis may be that local fibrin deposition could occur. Activation of coagulation driven by pulmonary inflammation [5] may be the trigger of a local widespread thrombotic event, thus inducing a life-threatening condition.

Surprisingly, our study also demonstrates a possible protective effect of hypertension. To the best of our knowledge, this association has never previously been described. It may be that patients with blood hypertension are less likely to develop severe hypotension at the time of an embolic event, thus increasing their chance of survival. Nevertheless, when hypertension and atrial fibrillation are concomitantly present, the risk of mortality increases. We suppose that hypertension may mitigate the low cardiac output in PE

TABLE 1 Variables included in univariate models and in the final logistic regression model associated with in-hospital mortality for pulmonary embolism

\begin{tabular}{|c|c|c|c|c|}
\hline Variables & \multicolumn{2}{|c|}{ Univariate } & \multicolumn{2}{|c|}{ Multivariate final model (stepwise) } \\
\hline Age $>75$ years & 0.005 & $2.316(1.215-4.402)$ & 0.005 & $2.424(1.301-4.517)$ \\
\hline Chronic obstructive pulmonary disease & 0.98 & $0.7189(0.2620-1.9723)$ & & Not included \\
\hline Diabetes & 0.91 & $0.863(0.320-2.327)$ & & Not included \\
\hline Atrial fibrillation & 0.1 & $1.34(0.627-2.870)$ & & Not included \\
\hline Hypertension & 0.04 & $0.393(0.184-0.839)$ & 0.004 & $0.266(0.109-0.648)$ \\
\hline Deep vein thrombosis & 0.001 & $0.099(0.0134-0.733)$ & 0.021 & $0.095(0.013-0.701)$ \\
\hline Stroke & 0.04 & $3.258(0.522-20.34)$ & & Not included \\
\hline Hypertension by atrial fibrillation ${ }^{\#}$ & 0.06 & $4.624(0.909-23.523)$ & 0.038 & $5.354(1.099-26.081)$ \\
\hline
\end{tabular}

\# : Logistic regression model. 
on one hand, while atrial fibrillation, by means of a basic reduction in cardiac output, may reverse this positive effect on the other. This possible effect may explain why the association between hypertension and atrial fibrillation reached a significant value.

In our study the presence of atrial fibrillation alone showed no association with mortality from PE, confirming some other reports [6], but in contrast to the findings of other authors [7-9].

Other comorbidities considered, such as diabetes and COPD, were not included in the final logistic regression model, thus indicating a negligible impact when present in terms of the risk for mortality from PE. However, a patient aged $>70$ years, who is hospitalised for a COPD exacerbation and is then forced to be bedridden presents an increased risk for VTE because of the high thromboembolic score.

Our study has some limitations. First, ICD-9-CM diagnosis codes are primarily used for reimbursement purposes and only secondarily for epidemiological research. They may be subject to potential sources of error, i.e. lack of validation for $\mathrm{PE}$, and in general are considered to have high specificity but only moderate sensitivity, thus being prone to underestimation of disease prevalence [10]. Secondly, we considered only patients who underwent imaging techniques (computed tomography and lung scan), thus limiting the sample size but avoiding unproven diagnosis of PE. A study like ours can help drive health policies in terms of decisions concerning resource allocation and improvement of prophylaxis and therapy. DVT and hypertension http://ow.ly/4nfs7v

Antonella Mameli ${ }^{1}$, Maria Antonietta Palmas ${ }^{2}$, Antonello Antonelli ${ }^{2}$, Paolo Contu ${ }^{3}$, Paolo Prandoni ${ }^{4}$ and Francesco Marongiu ${ }^{1}$

${ }^{1}$ Internal Medicine and Haemocoagulopathies Division, AOU of Monserrato, Monserrato, Italy. ${ }^{2}$ Dept of Health and Hygiene of the "Regione Autonoma della Sardegna" (RAS) Sardinian Regional Government, Cagliari, Italy. ${ }^{3}$ Dept of Public Health, University of Cagliari, Monserrato, Italy. ${ }^{4}$ Dept of Cardiovascular Sciences, University of Padua, Padua, Italy.

Correspondence: Antonella Mameli, Internal Medicine and Haemocoagulopathies Division, AOU, SS 554, 09042 Monserrato - Cagliari, Italy. E-mail: amameli@medicina.unica.it

Received: Nov 142015 | Accepted after revision: April 162016 | First published online: June 102016

Conflict of interest: None declared.

\section{References}

1 Konstantinides SV, Torbicki A, Agnelli G, et al. 2014 ESC guidelines on the diagnosis and management of acute pulmonary embolism. Eur Heart J 2014; 35: 3033-3069.

2 Kelly J, Hunt BJ, Rudd A, et al. Pulmonary embolism and pneumonia may be confounded after acute stroke and may co-exist. Age Ageing 2002; 31: 235-239.

3 Nijkeuter M, Söhne M, Tick LW, et al. The natural course of hemodynamically stable pulmonary embolism: clinical outcome and risk factors in a large prospective cohort study. Chest 2007; 131: 517-523.

4 Weberová $\mathrm{D}$, Weber $\mathrm{P}$, Kubesová $\mathrm{H}$, et al. Occurrence of pulmonary embolism among 260 in-patients of acute geriatric department aged 65+ years in 2005-2010. Adv Gerontol 2012; 25: 506-512.

5 van Langevelde K, Srámek A, Vincken PW, et al. Finding the origin of pulmonary emboli with a total-body magnetic resonance direct thrombus imaging technique. Haematologica 2013; 98: 309-315.

6 Koracevic G, Atanaskovic V. Is atrial fibrillation a prognosticator in acute pulmonary thromboembolism? Med Princ Pract 2010; 19: 166.

7 Sørensen HT, Horvath-Puho E, Lash TL, et al. Heart disease may be a risk factor for pulmonary embolism without peripheral deep venous thrombosis. Circulation 2011; 124: 1435-1441.

8 Prandoni P, Pesavento R, Sørensen HT, et al. Prevalence of heart diseases in patients with pulmonary embolism with and without peripheral venous thrombosis: findings from a cross-sectional survey. Eur J Intern Med 2009; 20: 470-473.

9 Ogren M, Bergqvist D, Eriksson H, et al. Prevalence and risk of pulmonary embolism in patients with intracardiac thrombosis: a population-based study of 23796 consecutive autopsies. Eur Heart J 2005; 11: 1108-1114.

10 O’Malley KJ, Cook KF, Price MD, et al. Measuring diagnoses: ICD code accuracy. Health Serv Res 2005; 40: $1620-1639$. 